\title{
SUSCEPTIBILIDADE DA MUCOSA GÁSTRICA À AÇÃO LESIVA DE UM SAL BILIAR EM RATOS CHAGÁSICOS CRÔNICOS
}

\author{
Lulz Ernesto de Almelda Troncon', Osman Cailxto Silva2, Sergio Zucoloto 3 , \\ Francisco Ferriolli Filho ${ }^{4}$ e Ulysses Garzella Meneghelli ${ }^{1}$
}

\begin{abstract}
Ratos inoculados previamente com a cepa $Y$ do Trypanosoma cruzi $(n=15) e$ ratos-controles de mesma idade e sexo $(n=15)$ foram submetidos a estudo que consistiu no exame da mucosa gástrica após a exposição in vivo da mesma à solução acidificada de um sal biliar, o taurocolato de sódio (TC). Em 10 animais de cada grupo procedeu-se também à contagem dos neurónios do plexo mientérico do estômago e ao exame histopatologico dos corpos neuronais, feito em microscopia optica convencional. Escores indicativos da gravidade das lesões produzidas pelo TC foram significativamente maiores $(P<0,002)$ nos ratos chagásicos que nos controles. A área erosada da mucosa gástrica, $\mathrm{em} \%$ da área total da região glandular do estômago, foi significativamente maior $(P<0,02)$ nos animais chagásicos $(25,2 \pm 18,0)$ que nos controles $(11,0 \pm 17,4)$. quanto ao número de neurônios intramurais do estômago, não foi observada diferença significante $(P>0,10)$ entre os ratos chagásicos $(1683,6 \pm 449,7 n=10)$ e os controles $(1732,8 \pm 401,1 n=10)$. Não houve diferença qualitativa ao exame histopatológico dos corpos neuronais. Estes resultados indicam que, na doença de Chagas experimental crônica, a mucosa gástrica é mais sensível à ação lesiva do $T C$, o que sugere redução da citoproteção natural do estómago. Esta anormalidade não deve decorrer de alterações da inervação intrínseca que impliquem redução do número de neurônios intramurais do estômago, mas pode ser o resultado de alterações qualitativas não detectáveis à microscopia óptica.
\end{abstract}

Palavras chaves: Doença de Chagas. Gastropatia chagásica. Mucosa gástrica. Úlcera gástrica. Sais biliares.

A forma crônica da doença de Chagas, que tem a miocardiopatia, o megaesôfago e o megacólon como manifestações clinicas mais freqüentes, constitui o resultado tardio da infecção pelo protozoário $T$. cruzi. $O$ substrato histopatológico da doença reflete a destruição de neurônios do sistema nervoso autônomo que, ao longo do tubo digestivo expressa-se por intensa redução do número das células ganglionares dos plexos nervosos intramurais ${ }^{17}$.

O comprometimento do estômago na doença de Chagas é reconhecido a partir das demonstrações da existência de destruição de neurônios intramurais do órgão, feitas tanto no homem ${ }^{211}$ como em animais de experimentação ${ }^{120}$. Ainda que apenas excepcionalmente resulte em manifestações clínicas próprias ${ }^{25}$, a lesão dos plexos intramurais do estômago traz como

Endereço para correspondência: Dr. L.E.A. Troncon Departamento de Clínica Médica do Hospital das Clínicas 14048 Ribeirão Preto, SP, Brasil.

1. Departamento de Clínica Médica, Faculdade de Medicina de Ribeirão Preto (FMRP).

2. Departamento de Cirurgia, Ortopedia e Traumatologia, FMRP

3. Departamento de Patologia, FMRP

4. Departamento de Parasitologia, Microbiologia e Imunologia, FMRP

Recebido para publicação em 27/2/86. conseqüência alterações expressivas da motilidade ${ }^{18}$ 212234 e da secreção gástrica 2223323334 .

Outro aspecto do comprometimento do estômago na doença de Chagas refere-se à maior prevalência, sugerida em chagásicos crônicos, de lesões da mucosa gástrica, como úlcera péptica ${ }^{9}$ e a gastrite superficial26 34. A origem destas lesões tem sido atribuida a conseqüências de possível disfunção motora antropiloro-duodenal, como a estase gástrica ${ }^{9}$ e o refluxo biliar duodeno-gástrico ${ }^{26}$.

O objetivo do presente trabalho foi testar experimentalmente a hipótese de que na doença de Chagas ocorra maior susceptibilidade da mucosa gástrica à ação lesiva do taurocolato de sódio, um sal biliar, que tem sido responsabilizado como causador de úlcera gástrica $^{27}$ e de lesões superficiais do estômago ${ }^{24}$.

\section{MATERIAL E MÉTODOS}

\section{A) Animais}

Foram estudados 30 ratos albinos, da linhagem Wistar, com idade variando de 13 a 19 meses, provenientes do Biotério Geral da Faculdade de Medicina de Ribeirão Preto. Estes animais foram distribuidos em 2 grupos:

a) Grupo de chagásicos crônicos: composto por 15 ratos com peso variando de $230 \mathrm{~g} \mathrm{a} 450 \mathrm{~g}$ (mediana: 
$300 \mathrm{~g})$ que foram estudados de 12 a 18 meses após a infecção experimental pelo $T$. cruzi. Esta foi efetuada logo após o desmame dos animais, tendo os mesmos 25 a 30 dias de idade, por inoculação de sangue de ratos doadores em fase aguda da infecção pela cepa $Y$ do $T$. cruzi. Cada animal recebeu por via intraperitoneal cerca de $0,2 \mathrm{ml}$ (cerca de 1000 tripomastigotas/g) de sangue obtido do doador por punção cardiaca. Todos os animais desenvolveram a fase aguda da doença, com parasitemia comprovada.

b) Grupo controle: composto por 15 ratos com idade $e$ distribuição por sexo semelhantes a do grupo de animais chagásicos e com peso variando de $230 \mathrm{~g}$ a $300 \mathrm{~g}$ (mediana: $248 \mathrm{~g}$ ). Estes animais foram mantidos desde os 30 dias de idade nas mesmas condições ambientais que as empregadas para os ratos do grupo de chagásicos.

\section{B) Procedimento}

A mucosa gástrica dos animais de ambos os grupos foi exposta, em condiçōes experimentais padronizadas, a uma solução acidificada de taurocolato de sódio. Nas 24 horas precedentes ao teste, foram os animais mantidos em jejum, em gaiolas individuais de fundo telado, para evitar a ingestão de pêlos e de fezes. Durante este periodo, os animais ingeriram "ad libitum" somente solução de sacarose a $10 \%$.

A técnica de estudo empregada baseou-se no conjunto de procedimentos descrito por Shay e colaboradores para produção experimental de úlceras ${ }^{29} \mathrm{e}$ para estudo da secreção gástrica ${ }^{30}$. Imediatamente antes do teste os animais eram pesados e, a seguir, anestesiados por administração intraperitoneal de solução a $25 \%$ de uretana, na dose de $0,5 \mathrm{ml} / 100 \mathrm{~g}$ de peso corporal. Em seguida, os ratos eram fixados em suporte apropriado. Estando os animais completamente anestesiados, procedia-se à abertura da cavidade abdominal por incisão mediana, seguida de exposição completa do estômago, ligadura total do piloro com fio de algodão e reparo do esôfago e dos nervos vagos anterior e posterior com outro fio de algodão. Neste momento, era introduzida sonda de polietileno de calibre adequado, pela boca do animal até o estômago. Por esta sonda era injetada solução $2,5 \mathrm{mM}$ de taurocolato de sódio em ácido clorídrico $0,1 \mathrm{~N}$, na dose de 2,0 ml/100 $\mathrm{g}$ de peso corporal. Imediatamente após o término da instilação era efetuada a ligadura total do esôfago, logo acima à transição esôfago-gástrica, realizada com o fio de algodão que separava os nervos vagos do corpo do esôfago, com o cuidado de não incluí-los na ligadura. Exatamente 1 hora após o término da instilação da solução do sal biliar, o estômagó era ressecado e aberto completamente ao longo da grande curvatura com tesoura de ponta fina e, a seguir, estirado por meio de fixação de suas margens com alfinetes, o suficiente para que as pregas da muco- sa fossem desfeitas. Assim preparado foi colocado em çuba contendo formalina a $10 \%$, com a mucosa voltada para a superficie líquida por periodo de pelo menos 24 horas.

\section{C) Avaliação da lesão da mucosa gástrica}

Estando o estômago fixado, colocava-se sobre o mesmo uma placa de vidro recoberta por papel fino, o que permitia que se delineasse, por transparência, os limites externos da área glandular, bem como os contornos das erosões produzidas.

Os desenhos obtidos eram então examinados por um observador que, desconhecendo a que grupo pertencia cada animal, avaliava a gravidade das lesões. Para tanto foram empregados dois critérios. $\mathrm{O}$ primeiro deles baseou-se em um sistema de escores ${ }^{7}$ segundo o qual cada lesão menor que $1 \mathrm{~mm}$ recebia o escore 1, entre 1 e $2 \mathrm{~mm}$ de diâmetro recebia o escore 2 , entre 2 e $3 \mathrm{~mm}$ o escore 3 e acima de $3 \mathrm{~mm}$ o escore 4. Para cada animal era então calculado um escore total, pela soma dos escores referentes a cada lesão.

O segundo critério foi a medida da área lesada, expressa em porcentagem da área total da região glandular do estômago. As referidas áreas foram estimadas por superposição dos desenhos obtidos em papel fino, acima mencionados, em papel milimetrado.

\section{D) Contagem dos neurônios intramurais do estômago}

Os neurônios do plexo de Auerbach foram estimados em 10 animais de cada grupo. Para tanto foi ressecada uma faixa do estômago de cerca de $1 \mathrm{~cm}$ de largura em toda extensão da víscera, na região glandular junto à transição com o rúmen. Estas faixas foram incluidas em parafina, para cortes seriados de $5 \mu \mathrm{m}$, tendo sido montado em lâmina um em cada cinco cortes. Assim, um total de $\mathbf{4 0}$ cortes seriados equivale em termos nas lâminas a $1 \mathrm{~mm}$ de espessura de cada animal ${ }^{1}$. Estes cortes foram corados pela hematoxilina-eosina. O escalonamento dos cortes evita a contagem dupla, visto que o diâmetro médio do neurônio mientérico em ratos é de $25 \mu \mathrm{m}^{4}$. Após a contagem dos neurônios, efetuou-se exame histopatológico sumário dos gânglios dos plexos nervosos intramurais

\section{E) Análise estatística}

A comparação dos resultados obtidos no grupo de ratos chagásicos com os dos controles foi analisada por meio do test " $U$ " de Mann-Whitney". Foram consideradas significantes as diferenças em que os valores de $\mathrm{p}$ foram inferiores a 0,05 .

\section{RESULTADOS}

Em todos os animais estudados, de ambos os grupos, foram observados edema e hiperemia da mucosa gástrica, coexistindo com número variável de petéquias e de erosões em áreas circunscritas, de ta- 
manho variável. Todas as lesões restringiam-se à região glandular do estômago, sendo poupado o rúmen (Fig. 1).

O exame macroscópico da mucosa gástrica após a exposição ao sal biliar revelou lesões mais acentuadas e mais extensas nos animais chagásicos que nos controles, conforme exemplifica a Figura 1.

Os escores indicativos da gravidade das erosões foram significativamente maiores $(\mathrm{p}<0,002)$ no grupo de ratos chagásicos que no controle. (Fig. 2).

Os valores referentes à área total das erosões da mucosa gástrica foram significativamente maiores $(\mathrm{p}<0,02)$ no grupo de animais chagásicos que entre os controles. (Fig. 3).

Assim sendo, a gravidade das lesões produzidas pelo sal biliar na mucosa gástrica dos animais com a doença de Chagas experimental, avaliada pelos 2 critérios, foi maior que no grupo-controle.

Quanto ao número de neurônios intramurais do estômago, (Fig. 4), não houve diferença estatisticamente significante $(p>0,10)$ entre os dois grupos de animais. Não se verificaram, também, diferenças entre chagásicos e controles quanto ao aspecto microscópico dos corpos celulares neuronais dos gânglios intramurais.

\section{DISCUSSÃO}

No presente estudo, demonstrou-se que ratos com doença de Chagas experimental crônica apresentam lesão mais grave da mucosa gástrica quando submetidos, em condições padronizadas, à ação lesiva de um sal biliar, o taurocolato de sódio.

Módena ${ }^{19}$, estudando a incidência de erosões agudas da mucosa gástrica conseqüentes à ligadura total do piloro seguida de indução de hipoglicemia insulínica em ratos chagásicos crônicos e controles verificou que mais de $95 \%$ dos primeiros desenvolviam lesões, o que ocorreu em menos de $15 \%$ dos últimos. Em ambos os grupos, as erosões restringiram-se à região glandular do estômago, como foi verificado neste trabalho. Estes resultados, tomados em conjunto com os do presente estudo, indicam que a mucosa secretora do estômago de animais chagásicos é mais susceptivel à lesão.

A proteção da mucosa do estômago, contra autodigestão cloridro-péptica e a ação de agentes lesivos, depende da integridade de vários fatores que, em conjunto, compõe a chamada "barreira da mucosa gástrica"12 14. Integram esta barreira, entre outros elementos, a estrutura lipoprotéica da membrana celular das células do epitélio gástrico, a camada fixa de muco que recobre este epitélio e a secreção alcalina de muco e de bicarbonato das células não parietais da mucosa do estômago ${ }^{14}{ }^{15}$. $\mathrm{O}$ álcool, antiinflamatórios não esteróides como a aspirina e a indometacina e os sais biliares são agentes capazes de provocar a ruptura da

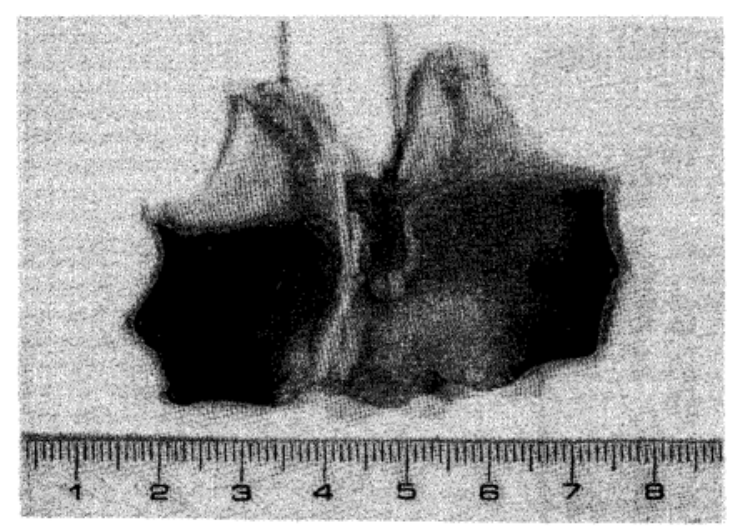

Fig. 1 - Lesões da mucosa gástrica produzidas pela exposição da mesma "in vivo" à solução acidificada do sal biliar em aninual chagásico (Esquerda) e controle (Direita). As lesōes, petéquias e erosões circunscritas, restringem-se à região glandular do estômago, sendo mais acentuadas no animal chagásico.

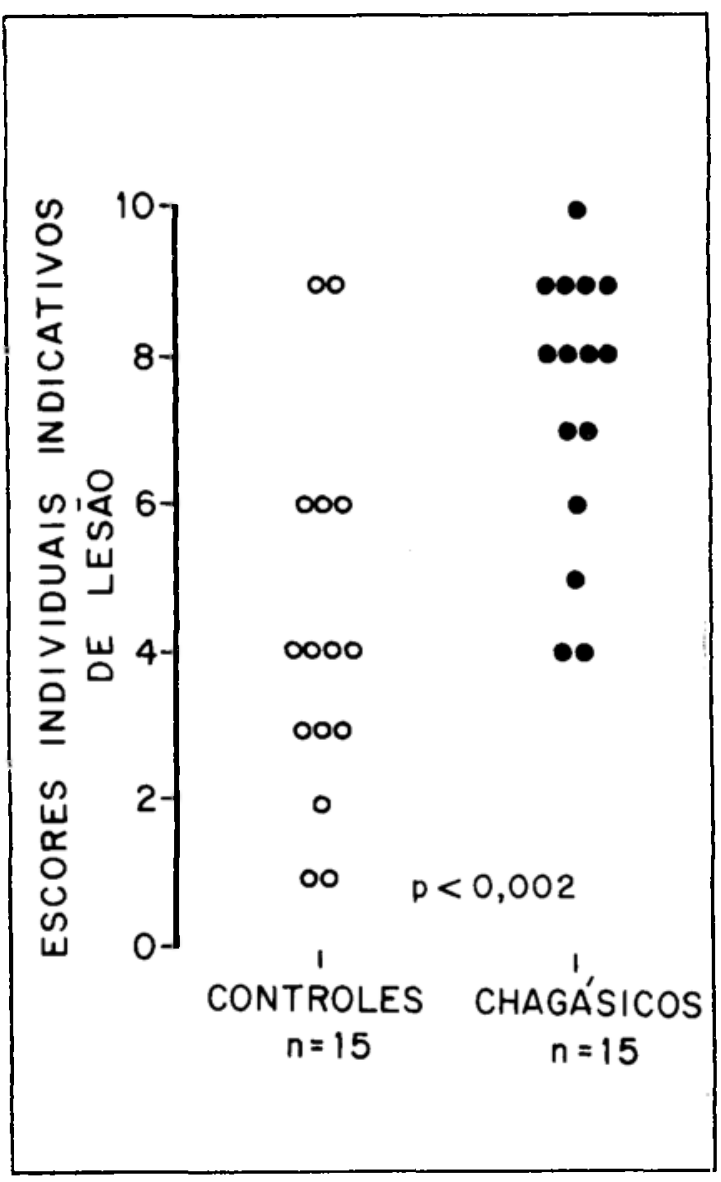

Fig. 2 - Distribuição dos escores individuais indicativos da gravidade das lesões da mucosa gástrica provocadas pela exposição ao taurocolato de sódio em ratoscontrole e chagásicos crônicos. 


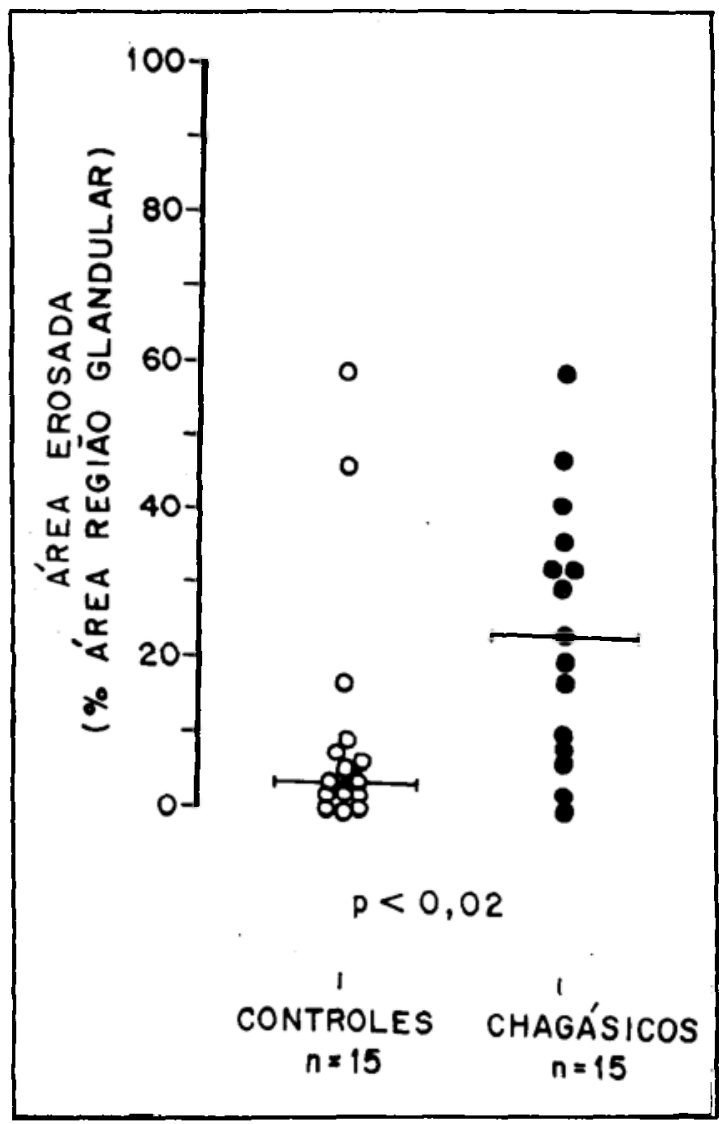

Fig. 3 - Distribuição dos valores referentes à área erosada da mucosa gástrica em ratos-controle e chagásicos crônicos. As barras horizontais indicam a mediana dos valores apresentados.

mencionada barreira, permitindo a retrodifusão de ácido e a conseqüuente lesão da mucosa gástrica ${ }^{12} 1415$. A administração prévia de prostaglandinas previne contra o desenvolvimento destas lesões ${ }^{28}$ efeito este denominado de citoproteção da mucosa gástrica 1014 16. O mecanismo responsável por esta ação citoprotetora não é perfeitamente conhecido, mas tem sido atribuído a algumas ações demonstradas das prostaglandinas como a redução da secreção gástrica de ácido e a estimulação da secreção alcalina de muco e de bicarbonato 131416 .

Algumas evidências de ordem experimental sugerem a importância de influências neurais na citoproteção da mucosa gástrica. Assim é que a estimulação vagal direta e a administração de drogas colinérgicas aumentam o conteúdo tissular de prostaglandinas na mucosa gástrica ${ }^{3}{ }^{16}$, bem como incrementam a secreção de muco e de bicarbonato pelo estômago ${ }^{13}$. Assim sendo, é de se supor que o bloqueio neural possa resultar em redução da eficiência da citoproteção. Em animais vagotomizados, entretanto, ocorre menor

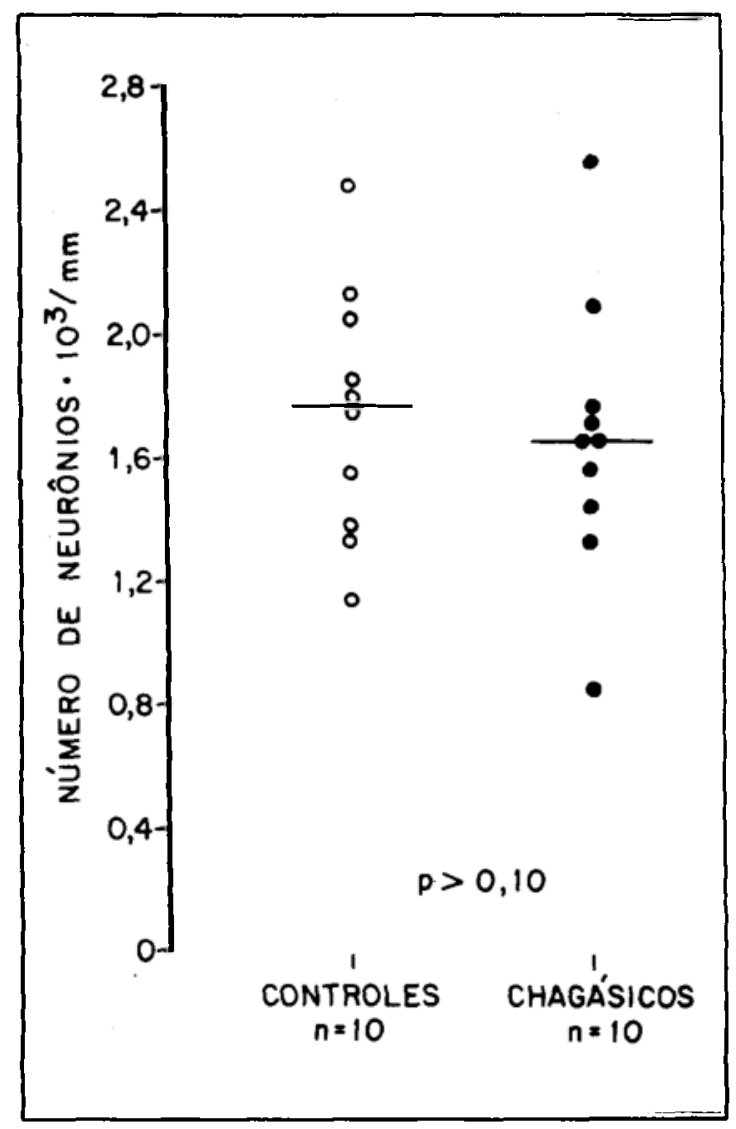

Fig. 4 - Resultado da contagem dos neurônios do plexo mientérico do estômago de ratos-controle e chagásicos crônicos. As barras horizontais indicam a mediana dos valores apresentados.

agressão à mucosa gástrica quando exposta a agentes lesivos $^{56}$. Nestas condições, é possível que o efeito da vagotomia em reduzir a secreção gástrica de ácido, o que tem ação citoprotetora específica ${ }^{7}$, sobreponha-se ao efeito da desnervação sobre os diferentes componentes da barreira da mucosa gástrica. Por outro lado, em ratos com doença de Chagas não tem sido possível demonstrar redução da secreção gástrica de ácido ${ }^{819}$, diferentemente do que tem sido repetidamente demonstrado no homem 2223323334 . Face ao exposto, é nossa interpretação que em ratos chagásicos crônicos, mantidos os níveis de secreção gástrica de ácido, o efeito de desnervação sobre os diferentes componentes da barreira resulta em diminuição da resistência da mucosa gástrica à ação de agentes lesivos.

Surpreendentemente, no presente trabalho, não foi possivel demonstrar no grupo de ratos chagásicos crônicos a esperada redução do número de neurônios intramurais do estomago, diferentemente do observado por outros autores que empregaram a cepa $Y$ do $T$. cruzi $i$ na produção da doença de Chagas experimen- 
Tronco LEA, Silva OC, Zucoloto $S$, Ferrioli Fo $F$, Meneghelli UG. Susceptibilidade de mucosa gástrica à ação lesiva de um sal biliar em ratos chagásicos crônicos. Revista da Sociedade Brasileira de Medicina Tropical 20: 199-204, Out-Dez, 1987

tal e o mesmo método da contagem de neurônios ${ }^{18}$. Também não se verificaram alterações degenerativas das células dos gânglios dos plexos nervosos intramurais. É possivel, portanto, que, nos animais integrantes do grupo de ratos chagásicos crônicos do presente estudo, tenha ocorrido algum grau de lesão da inervação intrínseca do estômago mas não suficientemente intensa para resultar alterações qualitativas à microscopia óptica convencional ou em redução do número de células ganglionares dos plexos intramurais. Não é possível descartar, contudo, a possibilidade de que outros fatores de natureza desconhecida, não relacionados com a inervação intrínseca do estômago, mas de algum modo dependentes da inoculação prévia com formas sangüícolas do $T$. cruzi sejam os responsáveis pela redução da resistência da mucosa gástrica à ação lesiva do taurocolato de sódio.

Em conclusão, os resultados do presente trabatho indicam que a mucosa gástrica de ratos com doença de Chagas experimental crônica é mais susceptível à ação lesiva de um sal biliar, o que sugere redução da citoproteção natural do estômago. $O$ esclarecimento dos mecanismos responsáveis pelo mencionado aumento da susceptibilidade à lesão, bem como o papel da inervação intrínseca do estômago na citoproteção da mucosa gástrica na doença de Chagas merecem outros estudos mais aprofundados.

Independentemente da natureza dos mecanismos responsáveis pelo aumento da susceptibilidade da mucosa gástrica à lesão, a presente demonstração da ocorrência desta anormalidade em ratos chagásicos crônicos permite incluí-la entre as possíveis causas da maior prevalência de lesões da mucosa gástrica, como a úlcera péptica ${ }^{9}$ e as gastrites superficiais 2634 , que têm sido sugeridas em pacientes chagásicos.

\section{SUMMARY}

Rats inoculated with the $Y$ strain of Trypanosoma cruzi and control rats of the same age and sex were submitted to examination of the gastric mucosa exposed in vivo to an acidified solution of a bile salt, sodium taurocholate (TC). Neuronal counts in the myenteric plexus of the stomach were also performed for several animals in both groups. The scores indicating the severity of the lesions produced by TC were significantly higher $(P<0.002)$ in chagasic $(n=15)$ than in control rats $(n=15)$. The eroded area of the gastric mucosa, expressed as a percentage of the total area of the glandular region of the stomach, was significantly larger $(P<0.02)$ in the chagasic (25.2 \pm 18.0$)$ than in control (11.0 \pm 17.4$)$ rats. As to the number of intramural ganglion cells in the stomach, no significant difference $(P>0.10)$ was observed between chagasic (1683.6 $\pm 449.7, n=10)$ and control $(1732.8 \pm 401.1, n=10)$ animals. These results indicate that in experimental chronic Chagas' disease the gastric mucosa is more sensitive to the damaging action of TC, suggesting reduction of the natural cytoprotection of the stomach. This abnormality does not appear to be due to changes in intrinsic innervation that may imply a reduction of the number of intramural neurons of the stomach.

Key-words: Chagas' disease. Chagasic gastropathy. Gastric mucosa. Gastric ulcer. Bile salts.

\section{REFERÊNCIAS BIBLIOGRÁFICAS}

1. Alcântara FG, Costa RB, Mello de Oliveira JA. Desnervação dos plexos intramurais do trato digestivo em Rattus novergicus albinus chagásicos crônicos. Revista Brasileira de Medicina 21: 560-563, 1964.

2. Amorim M, Corrêa Netto A. Histopathologia e pathogenese do megaesophago e megarecto (Considerações em tomo de um caso de "Mal de engasgo"). Annais da Faculdade de Medicina de São Paulo 8: 101-127, 1932.

3. Bennett A, Friedman CA, Vane JR. Release of prostaglandin $E_{1}$ from the rat stomach. Nature 216: 873-876, 1967.

4. Brandão HJS. Moléstia de Chagas experimental. Estudo quantitativo de neurônios parassimpáticos. O Hospital 61: 1013-1028, 1962.

5. Brodie DA. Ulceration of the stomach produced by restraint in rats. Gastroenterology 43: 107-109, 1962.

6. Brodie DA, Chase BJ. The role of aspirin-induced gastric irritation in the rat. Gastroenterology 53:604-610, 1967.

7. Carmichael HS, Nelson LM, Russel RI. Cimetidine and prostaglandin: evidence for different modes of action on the rat gastric mucosa. Gastroenterology 74: 1229-1232, 1978.

8. Ceneviva $R$. Aspectos anatomofuncionais do estômago relativo à úlcera gástrica aguda induzida experimentalmente em ratos normais, chagásicos crônicos e não-chagásicos vagotomizados. Tese de Livre-Docência, Faculdade de Medicina de Ribeirão Preto - USP, Ribeirão Preto, SP, 1972.

9. Ceneviva R, Módena JLP, Castelfranchi PL. Doença de Chagas e úlcera gástrica. Arquivos de Gastroenterologia de São Paulo 8: 85-88, 1971.

10. Chaudhury TK, Jacobson ED. Prostaglandin cytoprotection of gastric mucosa. Gastroenterology 74: 59-69, 1978.

11. Costa RB, Alcântara FG. Gastropatia chagásica crônica. Revista Brasileira de Medicina 22: 667-671, 1965.

12. Davenport HW. Physiological structure of the gastric mucosa. In: Code CF (ed). Handbook of Physiology, Alimentary canal. Vol. II, American Physiological Society, Washington, DC, pp. 759-779, 1967.

13. Feldman M. Gastric secretion. In: Slensenger $\mathbf{M H}$ \& Fordtran JS (ed). Gastrointestinal disease, WB Saunders Company, Philadelphia, pp. 541-577, 1983.

14. Fromm D. Gastric mucosal barrier. In: Johnson LR(ed). Physiology of the gastrointestinal tract. Vol. I, Raven Press, New York, pp. 733-748, 1983.

15. Garner A, Flemström G, Allen A. Current concepts of gastroduodenal mucosa protection. Scandinavian Journal of Gastroenterology 19 (Suppl. 92): 78-80, 1984. 
16. Johansson C, Bergstron S. Prostaglandins and protection of the gastroduodenal mucosa. Scandinavian Journal of Gastroenterology 17 (Suppl. 77): 21-46, 1982.

17. Köberle F. Chagas' disease and Chagas' syndromes. The pathology of American trypanosomiasis. Advances in Parasitology 6: 63-116, 1968.

18. Lopasso FP, Pinto Jr PE, Gama-Rodriguez JJ, Pinotti HW. Estudo do esvaziamento gástrico de partículas sólidas e digeriveis marcadas com $99 \mathrm{~m}$ Tc na gastropatia chagásica crônica. Gastroenterologia e Endoscopia Digestiva 4: 72-76, 1985.

19. Módena JLP. Alguns aspectos anatomofuncionais do estômago em ratos normais e chagásicos. Tese de Doutoramento, Faculdade de Medicina de Ribeirão Preto (USP), Ribeirão Preto, SP, 1972.

20. Okumura M, Corrêa Netto A. Produção experimental de "megas" em animais inoculados com Trypanosoma cruzi. Revista do Hospital das Clínicas 16: 338-341, 1961.

21. Oliveira RB, Troncon LEA; Meneghelli UG, Padovan W, Dantas RO, Godoy RA. Impaired gastric accomodation to distension and rapid gastric emptying in patients with Chagas' disease. Digestive Diseases and Sciences 25: 790-794, 1980.

22. Padovan W, Meneghelli UG, Godoy RA. Gastric secretory and motility studies in chronic chagasic patients. American Joumal of Digestive Diseases 22: 618-622, 1977.

23. Padovan W, Meneghelli UG, Godoy RA, Oliveira RB, Dantas RO, Troncon LEA. Acid and pepsin secretion in chronic chagasic patients in response to graded doses of pentagastrin and pentagastrin plus bethanecol. Digestion 23: 48-56, 1982.

24. Rees W, Rhodes J. Bile reflux in gastro-oesophageal disease. Clinics in Gastroenterology 6: 179-200, 1977.

25. Rezende JM. Manifestaçōes digestivas da doença de
Chagas. In: Dani R \& Castro LP (eds). Gastroenterologia Clínica. Vol. 2, Editora Guanabara Koogan SA, Rio de Janeiro, 1141-1168, 1981.

26. Rezende JM, Rosa H, Vaz MGM, Andrade-Sá N, Porto JD, Neves Neto J, Ximenez JAA. Endoscopia no megaesôfago: estudo prospectivo de 600 casos. Arquivos de Gastroenterologia de São Paulo 22: 53-62, 1985.

27. Rhodes J, Bernardo DE, Phillips SF, Rovelstad RA, Hoffman AF. Increased reflux of bile into stomach in patients with gastric ulcer. Gastroenterology 57: 241252, 1969.

28. Robert A. Effects of prostaglandins on the stomach and the intestine. Prostaglandins 6: 523-532, 1974.

29. Shay H, Komarov SA, Fels SS, Meranze D, Gruenstein M, Siplet H. A simple method for the uniform production of gastric ulceration in the rat. Gastroenterology 5: 43$61,1945$.

30. Shay H, Sun DCH, Erventein M. A quantitative method for measuring spontaneous gastric secretion in the rat. Gastroenterology 26: 906-913, 1954.

31. Siegel S. Non-parametric statistics for the behavioral on sciences. McGraw-Hill, New York, pp. 116-127, 1956.

32. Troncon LEA, Oliveira RB, Meneghelli UG, Dantas RO, Godoy RA. Fasting and food-stimulated plasma gastrin levels in chronic Chagas' disease. Digestion 29: 171-176, 1984.

33. Troncon LEA, Oliveira RB, Meneghelli UG, Dantas RO, Godoy RA. Plasma gastrin and gastric acid responses to insulin hypoglycemia in Chagas' disease. Brazilian Journal of Medical and Biological Research 18: 273278, 1985.

34. Vieira CB. Moléstia de Chagas e secreção gástrica. Bases para a individualização clínica da gastropatia chàgásica crônica. Tese de Livre-Docência, Faculdade de Medicina de Ribeirão Preto (USP), Ribeirão Preto, SP, 1968 . 